\title{
ON THE DIMENSION OF CERTAIN TOTALLY DISCONNECTED SPACES
}

\author{
LEX G. OVERSTEEGEN AND E. D. TYMCHATYN
}

(Communicated by James E. West)

\begin{abstract}
It is well known that there exist separable, metrizable, totally disconnected spaces of all dimensions. In this note we introduce the notion of an almost 0 -dimensional space and prove that every such space is a totally disconnected subspace of an R-tree and, hence, at most 1-dimensional. As applications we prove that the spaces of homeomorphisms of the universal Menger continua are 1-dimensional and that hereditarily locally connected spaces have dimension at most two.
\end{abstract}

\section{INTRODUCTION}

All spaces are separable and metric. Let $X$ be a space. The quasicomponent of a point $x \in X$ is the intersection of all subsets of $X$ which are both open and closed and which contain $x$. A space is totally disconnected provided each quasicomponent is a point. Compact, Hausdorff, totally disconnected spaces are 0 -dimensional. However, it is well known that there exist even complete, separable, totally disconnected, metric spaces of all dimensions.

A space $X$ is 0 -dimensional (resp., almost 0 -dimensional) provided there exists a basis $\mathscr{B}$ for $X$ such that for each $B \in \mathscr{B}, X \backslash \bar{B}$ is a clopen set (resp., $X \backslash \bar{B}$ is a union of clopen sets). Note that each 0-dimensional space is almost 0 -dimensional and that each almost 0 -dimensional space is totally disconnected. The set of points in Hilbert space, all of whose coordinates are irrational, is an example of a 1-dimensional, almost 0 -dimensional, totally disconnected space.

We prove that almost 0 -dimensional separable metric spaces are embeddable in $\mathbf{R}$-trees and, hence, have dimension bounded above by one. We will give two applications of this result; other applications will appear in a later note.

First we show that the spaces of homeomorphisms of the universal Menger continua are 1-dimensional. This extends a well-known result of Brechner [4], who had shown that the dimension of these spaces is at least one. Next we provide a partial answer to a question of $\mathrm{R}$. Duda (1964) by proving that the

Received by the editors October 27, 1992; presented at the 1989 Spring Topology Conference, Knoxville, TN, March 1989.

1991 Mathematics Subject Classification. Primary 54H20; Secondary 34C35, 58F13.

Key words and phrases. Totally disconnected, dimension, R-tree, Menger continua, homeomorphism groups, hereditarily locally connected.

The second named author was supported in part by NSERC grant A5616. 
dimension of a hereditarily locally connected, separable, metric space does not exceed two.

Let $X$ be a metric space with a given metric $d$. We will denote by $B(x, \varepsilon)$ the open ball of radius $\varepsilon$ around $x$ and by $\widehat{B}(x, \varepsilon)$ the closed ball of radius $\varepsilon$ around $x$. We will call a set which is both open and closed, clopen. For basic results and definitions which are not given here the reader may consult Kuratowski [6] or Whyburn [15].

\section{MAIN THEOREM}

A space is said to be arcwise connected if each pair of its distinct points is contained in an arc in the space (i.e., a subset of the space which is homeomorphic to the closed unit interval). A space is uniquely arcwise connected if it is arcwise connected and if it does not contain a homeomorphic copy of the unit circle in the plane.

A space $X$ is said to be an $\mathbf{R}$-tree if it is connected, locally arcwise connected, and uniquely arcwise connected. It is known (see [10]) that a nondegenerate $\mathbf{R}$-tree has dimension one. Every connected subset of an R-tree is an R-tree. A compact R-tree is called a dendrite. A point $x \in X$ is said to be an end-point of $X$ if $x$ is an end-point of each arc in $X$ which contains $x$. It is easy to see (see [10]) that the set of end-points of an R-tree is totally disconnected.

We shall prove that a space $X$ is almost 0 -dimensional if and only if $X$ is homeomorphic to the set of end-points of some R-tree.

Lemma 1. Let $X$ be almost 0-dimensional with respect to the basis $\mathscr{B}$. Then for each $B, C \in \mathscr{B}$ such that $\bar{B} \cap \bar{C}=\varnothing$, there exist disjoint clopen sets $H$ and $K$ in $X$ such that $X=H \cup K, B \subset H$, and $C \subset K$.

Proof. Suppose $X$ is almost 0 -dimensional with respect to the basis $\mathscr{B}$ and $B, C \in \mathscr{B}$ have disjoint closures. Then $X \backslash \bar{B}=\bigcup H_{n}$ and $X \backslash \bar{C}=\bigcup K_{m}$, where $\left\{H_{n}\right\}$ and $\left\{K_{m}\right\}$ are families of pairwise disjoint clopen sets. Consider

$$
G_{n}=H_{n} \backslash \bigcup\left\{K_{1}, \ldots, K_{n}\right\},
$$

and let $F=\bigcup G_{n}$. Then each $G_{n}$ is clopen, $C \subset F$, and $F \cap B=\varnothing$. It remains to be shown that $F$ is clopen. Since $F$ is a union of open sets, it is clearly open. Hence suppose $x \in \bar{F}$. Suppose first that $x \in \bar{C}$. Then $x \notin K_{m}$ for all $m$ and $x \in H_{n}$ for some $n$. Hence, $x \in G_{n} \subset F$. Assume next that $x \notin \bar{C}$. Then $x \in K_{m}$ for some $m$. Since $K_{m}$ is an open neighborhood of $x$ and $G_{n} \cap K_{m}=\varnothing$ for all $n \geq m, x \in \overline{U_{n<m} G_{n}}$. Since each $G_{n}$ is closed, $x \in G_{n}$ for some $n<m$ and $x \in F$. This completes the proof of the lemma.

Theorem 2. Let $X$ be an almost 0-dimensional space. Then $X$ embeds in (the set of end-points of ) an R-tree. In particular, $X$ is at most 1-dimensional.

Proof. Let $X$ be almost 0-dimensional with respect to the countable basis $\mathscr{B}=$ $\left\{B_{i}\right\}$. We will assume that $X$ is a subset of $Q \times\{0\} \subset Q \times I$, where $Q$ is the Hilbert cube with its usual metric $d$ and $I$ is the interval $[0,1]$.

The main idea of the proof is as follows: We will construct a uniquely arcconnected subset $R$ of $Q \times I$ such that $R \cap \pi^{-1}(0)=X$, where $\pi: Q \times I \rightarrow I$ is the natural projection. The space $R$ is then given a finer topology which makes it an R-tree with $X$ as a closed set of end-points. 
Let $\mathscr{U}_{1}=\{X\}, x_{X, 1} \in B_{1}, X_{1}=\left\{x_{X, 1}\right\}$; and let $T_{1}$ be the arc $\{r\} \times I$ where $x_{X, 1}=(r, 0)$. Then $x_{X, 1}$ is an end-point of $T_{1}$.

Suppose $\mathscr{U}_{n} \prec \mathscr{U}_{n-1} \prec \cdots \prec \mathscr{U}_{1}$ are finite covers of $X$ of minimum cardinality by pairwise disjoint clopen sets such that if $1 \leq r<s \leq i \leq n$ and $\bar{B}_{r} \cap \bar{B}_{s}=\varnothing$, then no element of $\mathscr{U}_{i}$ meets both $B_{r}$ and $B_{s}$. Hence, each element $U \in \mathscr{U}_{n}$ meets at least one of $\left\{B_{1}, \ldots, B_{n}\right\}$. Suppose $X_{1} \subset X_{2} \subset$ $\cdots \subset X_{n}$ are subsets of $X$ such that for each $i \leq n, X_{i}$ meets each $U \in \mathscr{U}_{i}$ in exactly one point $x_{U, i}$ and if $x_{U, i} \in X_{i} \backslash X_{i-1}$, then $x_{U, i} \in B_{j}$ for some $j \leq i$ such that $\operatorname{diam}\left(B_{j}\right)$ is minimal among $\left\{B_{k} \mid k \leq i\right.$ and $\left.B_{k} \cap U \neq \varnothing\right\}$. Suppose $T_{1} \subset T_{2} \subset \cdots \subset T_{n} \subset Q \times I$ are finite trees such that for $i \leq n, X_{i}$ is a set of end-points of $T_{i}$ and $T_{i} \cap \pi^{-1}(0)=X_{i}$. For $U \in \mathscr{U}_{i}$ let $K_{U, i}$ be a free arc in $T_{i}$ (i.e., $K_{U, i}$ minus its end-points is an open set in $T_{i}$ ) with end-point $x_{U, i}$ and so $K_{U, i} \cap K_{V, i}=\phi$ for $U \neq V$ in $\mathscr{U}_{i}$.

Suppose $\mathscr{U}_{n}, T_{n}$, and $X_{n}$ have been constructed. By Lemma 1, there is a finite cover $\mathscr{U}_{n+1}$ of $X$ of minimal cardinality of pairwise disjoint open sets in $X$ such that $\mathscr{U}_{n+1}$ refines $\mathscr{U}_{n}$ and such that if $1 \leq r<n+1$ and $\overline{B_{r}} \cap \overline{B_{n+1}}=\varnothing$, then no element of $\mathscr{U}_{n+1}$ meets both $B_{r}$ and $B_{n+1}$. If $U \in \mathscr{U}_{n+1}$ and $U \cap X_{n}=\varnothing$, let $x_{U, n+1} \in B_{i} \cap U$ where $1 \leq i \leq n+1$ and $B_{i}$ has minimal diameter among $\left\{B_{j} \mid j \leq n+1\right.$ and $\left.B_{j} \cap U \neq \varnothing\right\}$. If $U \in \mathscr{U}_{n+1}$ and $U \cap X_{n} \neq \varnothing$, let $W \in \mathscr{U}_{n}$ such that $U \subset W$ and let $x_{U, n+1}=x_{W, n}$. Let $X_{n+1}=\left\{x_{U, n+1} \mid U \in \mathscr{U}_{n+1}\right\}$.

Let $U \in \mathscr{U}_{n+1}$ and $W \in \mathscr{U}_{n}$ such that $U \subset W$. If $U \cap X_{n} \neq \varnothing$, let $A_{U, n+1}=\varnothing$ and if $U \cap X_{n}=\varnothing$, let $A_{U, n+1}$ be an irreducible piecewise linear arc from $x_{U, n+1}$ to $T_{n}$ such that $\pi \mid A_{U, n+1}$ is a homeomorphism, $A_{U, n+1}$ meets $K_{W, n}, A_{U, n+1} \cap A_{V, n+1}=\phi$ for $U \neq V$ in $\mathscr{U}_{n+1}, A_{U, n+1}$ is contained in the $2^{-n-1}$ ball around the straight line segment $\left[x_{U, n+1}, x_{W, n}\right]$ in $Q \times I$ and the length of the arc $A_{U, n+1}$ is less than $d\left(x_{U, n+1}, x_{W, n}\right)+2^{-n-1}$. Let

$$
T_{n+1}=T_{n} \cup \bigcup\left\{A_{U, n+1} \mid U \in \mathscr{U}_{n+1}\right\} .
$$

By induction $\mathscr{U}_{n}, T_{n}$, and $X_{n}$ are constructed for each positive integer $n$. For $y, z \in \cup T_{n}$ there is an integer $m$ such that $y, z \in T_{m}$. Let $[y, z]$ be the unique arc in $T_{m}$ from $y$ to $z$. Then $[y, z]$ is well defined and $X \cap[y, z] \subset\{y, z\}$. Note that $\bigcup T_{n} \backslash X$ is a locally finite simplicial complex which is a tree.

Let $R=X \cup\left(\cup T_{n}\right)$. We will first consider $R$ with the subspace topology and prove that in this topology $R$ is uniquely arcwise connected. Next we change the metric (and, hence, the topology) on $R$ such that it becomes locally arc connected. Since this new topology coincides with the subspace topology on arcs and on $X$, we will have completed the argument.

Choose a point $x \in X$. We will first show that there exists an arc in $R$ joining $x$ to $x_{X, 1}$. Next we will indicate how to modify the argument to show that given $\varepsilon>0$, there exists a neighborhood $B$ of $x$ in $X$ such that for each $y \in B$, there exists an arc $A$ in $R$ joining $x$ to $y$ such that $\operatorname{diam}(A)<\varepsilon$.

For each $n$ there exists a unique $U_{n} \in \mathscr{U}_{n}$ such that $x \in U_{n}$. Put $z_{n}=$ $x_{U_{n}, n}$. It suffices to show that $\lim z_{n}=x$ since in that case the subtree $T(x)$ spanned by $\{x\} \cup \bigcup\left\{z_{n}\right\}$ is a dendrite and, hence, a locally connected continuum. Each pair of points of $T(x)$ is contained in an arc in $T(x)$ which meets $X$ only in its end-points. Let $\left\{B_{i_{n}}\right\}$ be a sequence of basic sets containing $x$ 
such that $\operatorname{diam}\left(B_{i_{n}}\right) \leq 1 / n, B_{i_{1}}=X, \bar{B}_{i_{n}} \subset B_{i_{n-1}}$, and $i_{n-1}<i_{n}$.

It suffices to show that for each $n$ there exists an integer $a_{n}$ such that $z_{p} \in$ $B_{i_{n}}$ for each $p \geq a_{n}$. The proof is by induction on $n$. Let $a_{1}=1$, and suppose $a_{n-1}$ has been defined. Let $m>n$ be a positive integer such that $B\left(x, \frac{2}{m}\right) \subset B_{i_{n}}$. If $z_{q} \in B_{i_{n}}$ for each $q \geq i_{m}+a_{n-1}$ let $a_{n}=i_{m}+a_{n-1}$. Otherwise, let $q \geq a_{n-1}+i_{m}$ be smallest such that $z_{q} \notin B_{i_{n}}$. Since $\mathscr{B}$ is a basis for $X$, there is an integer $j \geq q$, such that $z_{q} \in B_{j}$, and $\bar{B}_{i_{m}} \cap \bar{B}_{j}=\phi$. Then $z_{q} \notin U_{j}$. Let $a_{n}$ be the smallest integer such that $z_{q} \notin U_{a_{n}}$. Then $q<a_{n} \leq j$. Then $z_{a_{n}-1}=z_{q}$. For $p \geq a_{n} \quad z_{p} \in B_{s}$ for some $s \in\{1, \ldots, p\}$ such that $\overline{B_{s}} \cap \bar{B}_{i_{m}} \neq \phi$ and $\operatorname{diam} B_{s} \leq \operatorname{diam} B_{i_{m}} \leq \frac{1}{m}$ since $x \in B_{i_{m}} \cap U_{p}$. It follows that $z_{p} \in B_{i_{n}}$.

Let $U=\bigcap\left\{B_{j} \mid j \leq a_{n}\right.$ and $\left.x \in B_{j}\right\}$. Let $y \in U \cap U_{a_{n}}$. For each positive integer $r$ let $V_{r}$ be the member of $\mathscr{U}_{r}$ such that $y \in V_{r}$ and let $\left\{y_{r}\right\}=V_{r} \cap T_{r}$. Then for $p \leq a_{n}, V_{p}=U_{p}$ and $y_{p}=z_{p}$. For $p \geq a_{n}, y_{p} \in B_{i_{n}}$ as above. The arc $\left[y, y_{a_{n}}\right]=\left[y, z_{a_{n}}\right]$ has diameter less than $\frac{2}{n}+2^{-a_{n}+1}$ since it lies by construction very close to the ball $B\left(x, \frac{1}{n}\right)$ in $Q$. It follows that each pair of points $y$ and $z$ in $U \cap U_{a_{n}}$ is contained in an $\operatorname{arc}[y, z] \subset(T(y) \cup T(z) \backslash X) \cup$ $\{y, z\}$ of diameter $<\frac{2}{n}+2^{-a_{n}+2}$.

Let $x, y \in X$ with $x \neq y$. Let $k$ be the smallest integer such that $x$ and $y$ lie in disjoint members $U$ and $V$, respectively, of $\mathscr{U}_{k}$. Each point $z$ of the nonempty set $\left[x_{U, k}, x_{V, k}\right] \backslash\left(K_{U, k} \cup K_{V, k}\right)$ separates $X \cup T_{k}$ between $U$ and $V$. By induction $z$ separates $X \cup T_{k+j}$ between $U$ and $V$ for each positive integer $j$. It follows that each pair of points $x$ and $y$ of $R$ is contained in a unique arc $[x, y]$ in $(R \backslash X) \cup\{x, y\}$.

Consider the following metric ${ }^{1}$ on $R$ :

$$
\rho(x, y)=\operatorname{diam}([x, y]) \text { for } x, y \in R \text {. }
$$

Then $\rho$ is clearly a metric which induces the same topology as the subspace topology on all arcs in $R$ and on the subspace $R \backslash X$. Hence $R$, in the topology induced by $\rho$, is still uniquely arcwise connected. By definition it is locally arcwise connected in this topology and, hence, an $\mathbf{R}$-tree. It remains to be shown that the topology induced on $X$ by $\rho$ coincides with the original topology (induced by the metric $d$ ) on $X$. Since $d(x, y)<\rho(x, y)$ for all $x, y \in X$, for each sequence $x_{n} \rightarrow x$ in the $\rho$ metric $x_{n} \rightarrow x$ in the $d$ metric. Hence assume $d\left(x_{n}, x\right) \rightarrow 0$. Let $\varepsilon>0$. By the above argument there exists a neighborhood $B$ of $x$ such that for each $y \in B$, there exists an arc of diameter $<\varepsilon$ joining $x$ to $y$. Hence $\rho\left(x_{n}, x\right)<\varepsilon$ for each $x_{n} \in B$. This completes the proof of the theorem.

\section{Applications}

Spaces of homeomorphisms. For each pair of positive integers $n$ and $k, n<$ $k$, Menger [11] has described an $n$-dimensional continuum $M_{k}^{n}$ which is universal with respect to containing homeomorphic copies of every $n$-dimensional compactum which can be embedded in $E^{k}$. For any $k>0, M_{k}^{0}$ is the Cantor set and $M_{2}^{1}$ is the Sierpinski universal plane continuum, and for $n \geq 1, M_{2 n+1}^{n}$

\footnotetext{
${ }^{1}$ Introduction of this metric simplified the original argument. We are indebted to John Mayer for this suggestion.
} 
is the $n$-dimensional Menger universal continuum. Alternative constructions and related results are given in $[3,5,7,14]$. Anderson [1] for $n=1$ and Bestvina [2] have provided elegant characterizations of the Menger universal continua and proved their homogeneity. Cannon [5] and Whyburn [15] characterized the $(n-1)$-dimensional Sierpinski Continua $M_{n}^{n-1}, n \neq 4$; and Lewis announced that $M_{k}^{n}$ is not homogeneous for $n>0$ and $k<2 n+1$. Let $\mathscr{H}_{k}^{n}$ denote the space of homeomorphisms of $M_{k}^{n}$ with the sup metric $d$. Brechner [4] has essentially shown that $\mathscr{H}_{2 n+1}^{n}$ is at least 1 -dimensional and totally disconnected. It was conjectured that this space is infinite dimensional. We will prove that it is almost 0 -dimensional and, hence, 1-dimensional. We first establish the following lemma.

Lemma 3. Let $X$ be a metric space, $x \in X$, and let

$$
N=\{\varepsilon>0 \mid \overline{B(x, \varepsilon)} \neq \widehat{B}(x, \varepsilon)\} .
$$

Then $N$ is at most countable.

Proof. Let $\mathscr{B}=\left\{B_{i}\right\}$ be a countable basis for $X$. Note that for each $\varepsilon \in N$, there exists a point $x_{\varepsilon} \in \widehat{B}(x, \varepsilon)$ and a number $\eta_{\varepsilon}>0$ such that

$$
B(x, \varepsilon) \cap B\left(x_{\varepsilon}, \eta_{\varepsilon}\right)=\varnothing .
$$

For each $\varepsilon \in N$, choose a basic set $B_{i_{\varepsilon}} \in \mathscr{B}$ such that $x_{\varepsilon} \in B_{i_{\varepsilon}} \subset B\left(x_{\varepsilon}, \eta_{\varepsilon}\right)$. If $N$ is uncountable, then there exists an index $n$ and $\varepsilon_{1}<\varepsilon_{2}$ in $N$ such that $i_{\varepsilon_{1}}=i_{\varepsilon_{2}}=n$. Then $x_{\varepsilon_{1}} \in B_{n} \subset B\left(x_{\varepsilon_{2}}, \eta_{\varepsilon_{2}}\right)$. This contradicts (1) and completes the proof of the lemma.

Theorem 4. Let $X$ be a metric space and $D$ a dense set of points of $X$. Suppose that for each $d \in D$ there exists a dense $G_{\delta}$ set $E_{d}$ of positive numbers such that for each $\varepsilon \in E_{d}, X \backslash \widehat{B}(d, \varepsilon)$ is a union of clopen sets. Then $X$ is almost 0-dimensional.

Proof. By Lemma 3, for each $d \in D$, there exists a dense $G_{\delta}, F_{d} \subset E_{d}$, such that $\overline{B(d, \varepsilon)}=\widehat{B}(d, \varepsilon)$ for each $\varepsilon \in F_{d}$. Hence, the result follows immediately.

Theorem 5. $\mathscr{X}_{k}^{n}$ is almost 0-dimensional (and, hence, at most 1-dimensional) for $k \geq n+1$.

Proof. By Theorem 4, it suffices to show that the complement of each closed ball in $\mathscr{X}_{k}^{n}$ is a union of clopen sets. Hence fix $\mathscr{H}_{k}^{n}(k \geq n+1), g \in$ $\mathscr{H}_{k}^{n}$, and $\varepsilon>0$. We shall show that for each $h \in \mathscr{X}_{k}^{n}$ with $d(h, g)>\varepsilon$, there exists a clopen set $U$ in $\mathscr{X}_{k}^{n}$ containing $h$ such that $U \cap \widehat{B}(g, \varepsilon)=$ $\varnothing$. Suppose $d(h, g)=\varepsilon+4 \delta, \delta>0$, and $x \in M_{k}^{n}$ such that $d(h, g)=$ $d(h(x), g(x))$. Choose a $n$-sphere $S$ in $M_{k}^{n}$ such that $g(S) \subset B(g(x), \delta)$ and $h(S) \subset B(h(x), \delta)$. By Nagata [12], there exists a retraction $r: M_{k}^{n} \rightarrow h(S)$ such that $r\left(M_{k}^{n} \backslash B(h(x), 2 \delta)\right)=$ point $\}$. Let $U=\left\{f \in \mathscr{X}_{k}^{n}|r \circ f| S \not *\right\}$. Then $U$ is clopen in $\mathscr{H}_{k}^{n}$ since near maps into an ANR are homotopic. Also $h \in U$ and for each $f \in B(g, \varepsilon), f(S) \subset B(g(x), \varepsilon+\delta)$, so $r \circ f(S)$ is a point and $f \notin U$.

Corollary 6. The set of homeomorphisms $\mathscr{Z}_{k}^{s}$ of $M_{k}^{s}$ is 1-dimensional for $(s, k)$ $\in\{(n, 2 n+1) \mid n \geq 1\} \cup\{(n, n+1) \mid 1 \leq n \neq 3\}$.

Proof. A space $X$ is locally setwise homogeneous if there exists a basis $\mathscr{B}$ of connected open sets and a dense countable subset $D \subset X$ such that for 
any $B \in \mathscr{B}$ and any two points $x, y \in B \cap D$ there exists a homeomorphism $h: X \rightarrow X$ such that $h(x)=y$ and $h \mid X \backslash B=\mathrm{id}_{X \backslash B}$. Brechner [4] has shown that the group of homeomorphisms of any locally setwise homogeneous continuum is at least 1 -dimensional. Hence (cf. $[4,5,2]), \mathscr{H}_{k}^{s}$ is at least 1dimensional. By Theorems 5 and $2, \mathscr{H}_{k}^{s}$ is at most 1 -dimensional and the result follows.

Hereditarily locally connected spaces. A space is said to be hereditarily locally connected if it is connected and if each of its connected subsets is locally connected. Clearly, each $\mathbf{R}$-tree is hereditarily locally connected. We shall prove that each hereditarily locally connected space $X$ can be written as $X=Y \cup C$ where $Y$ is almost 0 -dimensional and $C$ is countable. It follows that the dimension of each hereditarily locally connected space does not exceed two. We recall two results from [13, p. 586 and p. 597] concerning hereditarily locally connected spaces.

Theorem 7. If $X$ is a hereditarily locally connected space and $Y$ is a subset of $X$, then the components of $Y$ are also the quasicomponents of $Y$.

Theorem 8. Let $X$ be a hereditarily locally connected space, $T$ a totally disconnected subset of $X$, and $Y$ a closed subset of $X$. Then the nondegenerate components of $Y \cup T$ are the nondegenerate components of $Y$.

The following corollary is of independent interest.

Corollary 9. If $Y$ is a totally disconnected subset of a hereditarily locally connected space $X$, then $Y$ is almost 0-dimensional.

Proof. Let $\left\{V_{i}\right\}_{i=1}^{\infty}$ be a basis of connected open sets for $X$. For each positive integer $i$ let $U_{i}=V_{i} \cap Y$. Suppose $\bar{V}_{i} \cap \bar{V}_{j}=\varnothing$. By Theorem $8, \bar{V}_{i}$ and $\bar{V}_{j}$ are components of $Y \cup \overline{V_{i} \cup V_{j}}$. Hence, $Y$ can be separated between $U_{i}$ and $U_{j}$ by Theorem 7 .

It is known [13, p. 573] that there is an R-tree with a 1-dimensional set $E$ of end-points. By Corollary $9, E$ is almost 0 -dimensional. The set of irrational sequences in the Hilbert space $l_{2}$ and the set of end-points of the universal separable R-tree [8] are other examples. (By a recent announcement of Fokkink and Oversteegen, see [9], these spaces are in fact homeomorphic.)

The following corollary follows immediately from Corollary 9 and Theorem 2.

Corollary 10. A space $X$ is almost 0-dimensional if and only if it can be embedded in the set of end-points of an $\mathbf{R}$-tree.

It is a classical result of Whyburn (see [15, p. 94]) that a compact hereditarily locally connected space $X$ has a basis of open sets with countable boundaries and, hence, has dimension one. In [13, Theorem 2.4] Whyburn's theorem was improved by replacing compactness of $X$ by semicolocal connectedness of $X$. (A space $X$ is semicolocally connected if $X$ has a basis of open sets $\left\{V_{i}\right\}_{i=1}^{\infty}$ such that for each $i \quad X \backslash V_{i}$ has finitely many components.) In [13, p. 593] it was shown (by constructing an $\mathbf{R}$-tree $T$ with a set of end-points which is not rim-countable) that semicolocal connectedness is necessary in that result. R. Duda posed the following (see $[13, \S 5]$ ): 
Problem 1. If $X$ is a separable, metric, nondegenerate, hereditarily locally connected space, is $\operatorname{dim}(X)=1$ ? lem.

The following theorem provides a partial affirmative solution to Duda's prob-

Theorem 11. If $X$ is a separable, metric, hereditarily locally connected space, then $X=Y \cup C$ where $Y$ is almost 0-dimensional and $C$ is countable. In particular, $\operatorname{dim}(X) \leq 2$.

Proof. By [13, Theorem 2.3], $X=Y \cup C$ where $Y$ is a totally disconnected set and $C$ is a countable set. By Corollary $9, Y$ is almost 0-dimensional. By Theorem $2, \operatorname{dim}(Y) \leq 1$ and, hence,

$$
\operatorname{dim}(X) \leq \operatorname{dim}(Y)+\operatorname{dim}(C)+1 \leq 1+0+1=2 .
$$

\section{REFERENCES}

1. R. D. Anderson, A characterization of the universal curve and a proof of its homogeneity, Ann. of Math. (2) 67 (1958), 313-324.

2. Mladen Bestvina, Characterizing $k$-dimensional universal Menger compacta, Mem. Amer. Math. Soc., vol. 71, Amer. Math. Soc., Providence, RI, 1988.

3. H. G. Bothe, Universal mengen bzuglich der lage im $E^{n}$, Fund. Math. 56 (1964), 203-212.

4. Beverly Brechner, On the dimension of certain spaces of homeomorphisms, Trans. Amer. Math. Soc. 121 (1966), 516-548.

5. J. W. Cannon, A positional characterization of the $(n-1)$-dimensional Sierpinski curve in $S^{n}(n \neq 4)$, Fund. Math. 79 (1973), 107-112.

6. K. Kuratowski, Topology. II, Academic Press, New York, 1968.

7. S. Lefschetz, On compact spaces, Ann. of Math. 2 (1931), 521-538.

8. John C. Mayer, J. Nikiel, and L. G. Oversteegen, Universal spaces for R-trees, Trans. Amer. Math. Soc. 334 (1992), 411-432.

9. John C. Mayer and L. G. Oversteegen, Continuum theory, Recent Progress in General Topology (M. Hušek and J. van Mill, eds.), North-Holland, Amsterdam, 1992, pp. 453-492.

10. _ A topological characterization of R-trees, Trans. Amer. Math. Soc. 320 (1990), 320-395.

11. K. Menger, Kurventheorie, Teubner, Leipzig, 1932.

12. J. Nagata, Modern dimension theory, Wiley (Interscience), Groningen, 1965.

13. Togo Nishiura and E. D. Tymchatyn, Hereditarily locally connected spaces, Houston J. Math. 2 (1976), 581-599.

14. B. A. Pasynkov, Partial topological products, Trudy Moskov. Mat. Obshch. 13 (1965), 136-245; English transl., Trans. Moscow Math. Soc. (1965), 153-271.

15. G. T. Whyburn, Analytic topology, Amer. Math. Soc. Colloq. Publ., vol. 28, Amer. Math. Soc., Providence, RI, 1942.

University of Alabama at Birmingham, Department of Mathematics, Birmingham, AlABAMA 35294

E-mail address: overstee@math.uab.edu

University of Saskatchewan, Department of Mathematics, Saskatoon, Saskatoon, CANADA S7N OWO

E-mail address: tymchat@snoopy.usask.ca 